\title{
Simulated self-organization of death by inherited mutations
}

\author{
JORGE S. SÁ MARTINS ${ }^{1,2}$, DIETRICH STAUFFER ${ }^{1,3}$ \\ PAULO M.C. DE OLIVEIRA ${ }^{1,2}$ and SUZANA MOSS DE OLIVEIRA ${ }^{1,2}$ \\ ${ }^{1}$ Laboratoire PMMH, École Supérieure de Physique et de Chimie Industrielles \\ 10 rue Vauquelin, F-75231 Paris, France \\ ${ }^{2}$ Visiting from Instituto de Física, Universidade Federal Fluminense \\ Av. Litorânea s/n, Boa Viagem, 24210-340 Niterói, RJ, Brasil \\ ${ }^{3}$ Visiting from Institute for Theoretical Physics, Cologne University, D-50923 Köln, Euroland \\ Manuscript received on September 26, 2008; accepted for publication on April 1, 2009; \\ contributed by PAULO M.C. DE OLIVEIRA*
}

\begin{abstract}
An agent-based computer simulation of death by inheritable mutations in a changing environment shows a maximal population, or avoids extinction, at some intermediate mutation rate of the individuals. Our results indicate that death seems needed to allow for evolution of the fittest, as required by a changing environment.
\end{abstract}

Key words: changing environment, computer modeling, evolution, mutation, selection.

\section{INTRODUCTION}

More than a century ago, Weissmann argued that aging and death are needed to make place for our children; and children are, in turn, needed to allow for Darwinian evolution through survival of the fittest. Kirkwood (2005) summarized this theory of aging and many other ones, and specific computer models of aging and death were reviewed (Moss de Oliveira et al. 1999, Stauffer et al. 2001, 2006), such as the Penna model (Penna 1995, Moss de Oliveira et al. 1996, Stauffer 2007), and the oldest-old effect (de Oliveira et al. 1998, 1999). A mathematical argument against immortality was recently given in this sense (de Oliveira 2007a, 2009).

Now we want to understand the need for death through Monte Carlo simulations of individuals. We distinguish between newborns and adults, and take into account environmental changes. They may come from climate change, like ice ages and warmer periods during the existence of Homo sapiens, or they may be caused by

\footnotetext{
*Member Academia Brasileira de Ciências Correspondence to: Paulo Murilo Castro de Oliveira E-mail: pmco@if.uff.br
}

migrations of people from one environment to another. A single environmental change was already used to justify sexual over asexual reproduction (Sá Martins and Moss de Oliveira 1998) or to account for geographic variation (Cebrat and Pekalski 2004). Thus, we may allow the mutation rate of individuals to change in order to find its optimal value. Here "optimal" either means a maximum of the population in a fixed environmental carrying capacity, or survival instead of extinction, depending on which of our two models (A and B) were used.

In these two models, by using sexual reproduction, the genome is represented by two strings of $L$ bits each, i.e. $L$ loci. They represent the $L$ most serious genetic diseases. Each mutation damaging the phenotype (i.e. the health of the individual) reduces the survival probability per iteration by a factor $x$. As genetic load, we count those bit positions that differ from an ideal bit-string. This latter bit-string is initially zeroed, with changes happening at each iteration with a probability $\mathrm{p}$ at one randomly selected bit position. It thus represents the requirement of the changing environment at each locus. For reproduction, the two bit-strings of the father are 
crossed-over at one randomly selected bit position, the same happening for the mother, and then one of the two resulting bit-strings from the father (the gamete) is combined with one of the two from the mother to give the child genome. Mutations are also inherited from the parents, and $m$ new mutations are introduced at birth to each gamete (if $m \geq 1$; for $m<1$, one new mutation is added with probability $m$ ). The genetic load $N$ is the number of loci (bit positions) where the genome is not adapted (i.e., it is not the same as the ideal string) to the current environment. All the changes in the individuals and the environmental bit-strings are reversible.

Model A's population is not constant and finds as an optimal $m$ - a mutation rate for which the equilibrium population reaches a maximum. Model B follows a tradition of theoretical biology and keeps the population constant except if all adults die out during one iteration; then, we check which mutation rate avoids the extinction of the whole population. Further details of the two models will be discussed in the corresponding sections.

\section{MODEL A WITH CHANGING POPULATION}

In model $\mathrm{A}$, each of the individuals survives the next time step (iteration involving all survivors) with probability $x^{N}(1-P / K)$, in which $P$ is the current total population and $K$ is a fixed input parameter, sometimes called the carrying capacity, representing limitations [due to the lack of food and space] for the population growth. Here, $N$ is the genetic load, the number of bits that are not adapted to the current requirement of the environment. The Verhulst factor $1-P / K$ applies to all individuals, differently from Sá Martins and Cebrat (2000).

Recessiveness is defined differently in two different versions A1 and A2 of model A, implying in a different computational procedure to determine the genetic load $N$ in each one. For A1, the computational procedure is to take the logical and of the two bit-strings of the individual, and then count as $N$ the number of bit positions where the result of the and operation differs from the ideal string. This procedure is close to (Stauffer and Cebrat 2006), and means that heterozygous loci do not count for the genetic load $N$ if the ideal string (the environment) has a bit zero in those loci, but count if the bits are set. For A2, we count for $N$ only those positions where both individual bit-strings agree with each other (homozygous loci) and disagree with the ideal bit-string. In biological terms, for A1 the allele 0 is always dominant over the recessive allele 1 . For A2, in contrast, allele 1 becomes dominant and allele 0 recessive when the environmental bit is 1 instead of 0 .

Our $K$ is mostly 2 million, the initial population is $K / 5$, and the resulting equilibrium population is mostly of the order of one million if it does not die out. The two individual bit-strings are mutated independently, each one with a mutation rate $m$. Each surviving female adult at each iteration gives birth to $B$ babies, which become adult at the next iteration; we used $B=4$. Mostly 10,000 iterations were made $(100,000$ for most cases with $m<0.001$ ), and averages were taken from the second half of this time interval.

\section{CASE A1}

Figure 1 shows our main result: the population $P$ has a maximum as a function of $m$ at some intermediate $m$ value. Thus, neither very small $m$ ("eugenics") nor very large $m$ ("instability") are optimal; an intermediate mutation rate leads to the largest $P$ or the lowest $\langle N\rangle$, and, in all cases, to a finite lifespan.

Instead of applying the Verhulst deaths to all ages, Figure 2 shows the correlation between genetic deaths only and genetic load by applying the Verhulst deaths only to the births (Sá Martins and Cebrat 2000). Data are taken from averages calculated at different time steps of a single run for each value of $x$.

\section{CASE A2}

The modified recessiveness defined above for model A2 reduces $N$ and makes survival possible for a changing environment even for an unrealistically small $x$. For $x \geq$ 0.8 we see in Figure 3 top a plateau for small mutation rates $m$, followed by a decay for a larger $m$. Thus, there is no longer the clear population maximum as it was seen in model A1. A similar result was obtained for model A1 in a stable environment (not shown).

\section{MODEL B WITH CONSTANT POPULATION}

In this version, there are no random deaths by the Verhulst factor. Instead, individuals die exclusively due to genetic reasons. At each time-step, each individual with a genetic load $N$ survives with probability $x^{N+1}$. So, 

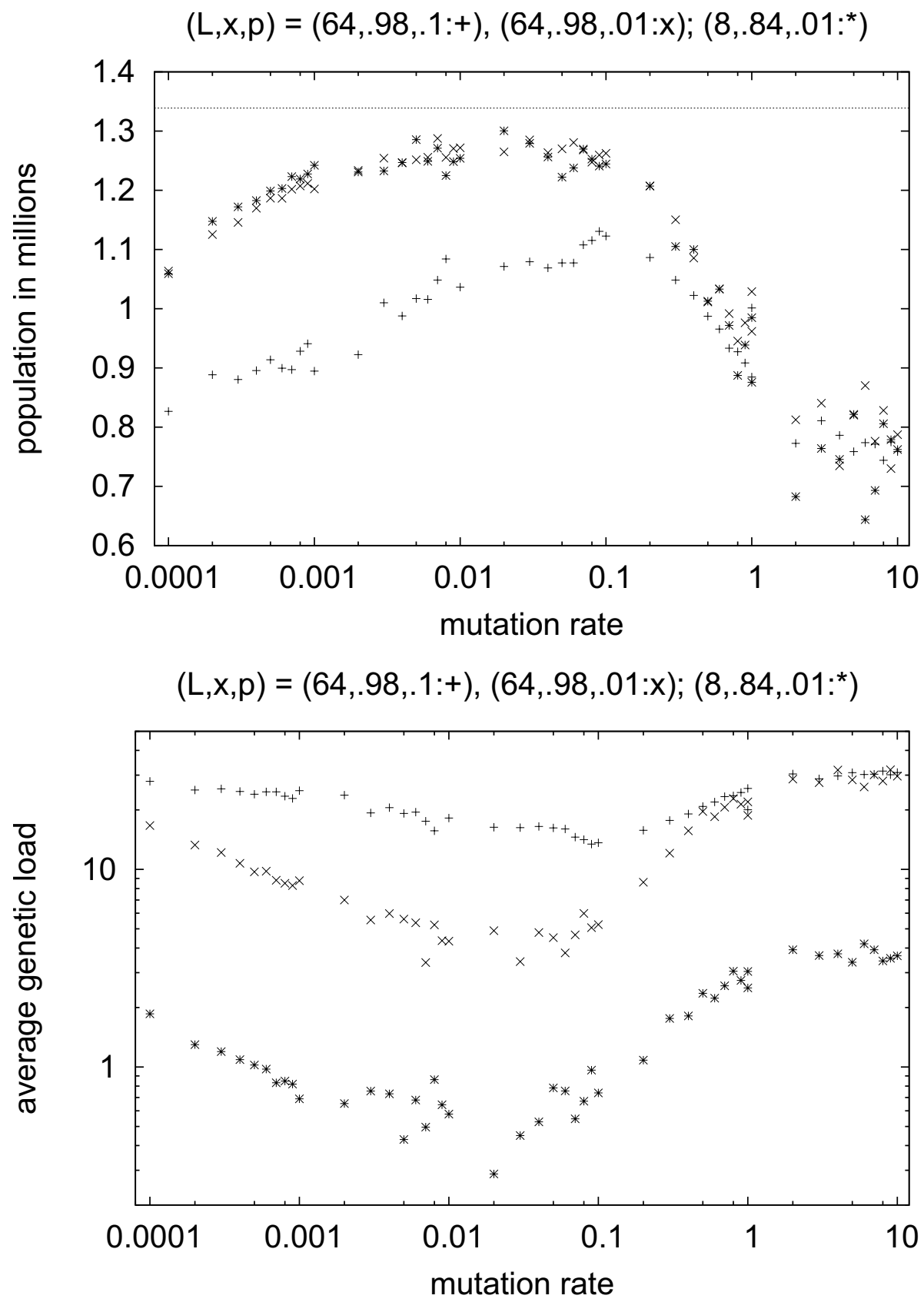

Fig. 1 - Model A1. Search for the optimal mutation rate, in which the population (top) reaches the maximal value and the number $\langle N\rangle$ of unadapted loci (genetic load, bottom) gets the minimal, at $x=0.98$. For $x=0.99, L=64, p=0.01$, the results are similar; for $x=0.96$, the populations die out for some values of these parameters.

an individual with zero load can still die with a probability $1-x$. This selection mechanism may lead to the extinction of the whole population for some values of the model's parameters. If there is no extinction at a given time step, the survivors breed, generating new individuals until the initial population size is restored for the next time step. There is no distinction between males and females, and the population may be regarded as one of hermaphrodites.

To generate the offspring's genome, the genetic strings of each parent are crossed-over and one gamete of each is randomly chosen. A number $M$ of muta- 


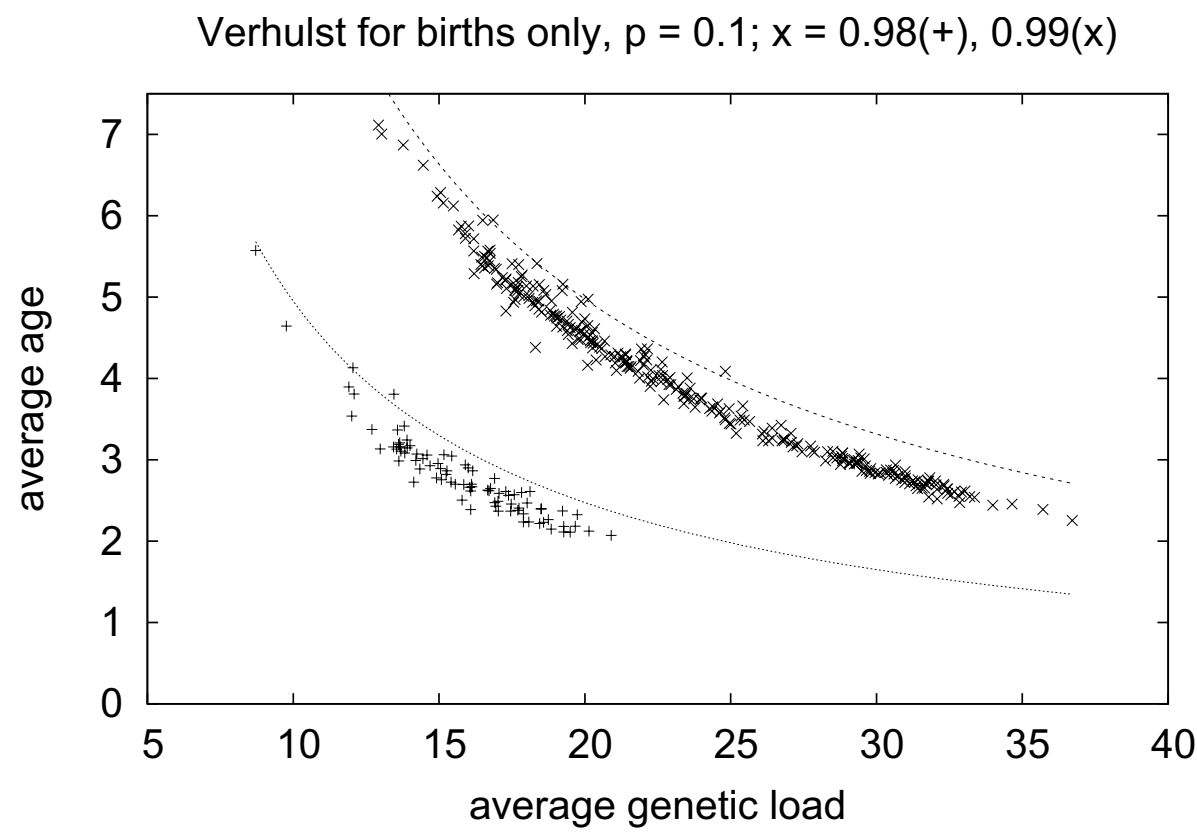

Fig. 2 - Model A1. Average age of survivors versus the number of unadapted loci, when the Verhulst death probability applies to the births only; the individuals are represented by pairs of 64-bit strings and results are shown for various values of $m$ after some simulation time. The curves show $1 /(|\ln x| N)$.

tions extracted from a uniform distribution in the interval $[0,2 m)$ is then introduced in this genome, each one at a random location of a randomly chosen gamete. Thus, $m=2$ in this model (B) corresponds to $m=1$ in model A. If $M$ is not an integer, then $\operatorname{int}(M)$ mutations are added, where $\operatorname{int}(x)$ is the largest integer contained in $\mathrm{x}$, and an extra mutation is added with probability $M-\operatorname{int}(M)$. As a result of this strategy, $m$ new mutations are added to each offspring genome on average.

The model treats heterozygous loci in the same way as model A2, that is, they never contribute to the genetic load. A slightly different version of this model, in which $x$ was recalculated at each time step to keep constant the fraction of deaths, was presented in de Oliveira (2001) and de Oliveira et al. (2008).

The results for this model shown in Figure 4 should be compared to Figure 1. We keep the mutation rate of the environment as $p=0.01$, the selection strength as a fixed $x=0.98$ and then compute the average genetic load $\langle N\rangle$, the fraction of the population that dies per time step, and the fraction of perfect, or ideal, genomes in the population for different values of the mutation rate $m$. (Perfect, here, means that no homozygous unadapted locus is present, although heterozygous loci can be present at the individual's genome). We find that there is an intermediate range of values of the mutation rate for which both the genetic load and the death rate go through minima, while the fraction of perfects reaches a maximum. This result matches with what was found, in similar situations, in our model A1. (For $L=3200$ in Fig. 4, extinction happens for mutation rates below 0.1 or above 1.2.).

Our main result refers to the need for a strong selection mechanism as a means to enforce a small genetic load: death of the least adapted individuals makes way to fitter ones. In Figure 5 we show the time evolution of the average genetic load of the population for four different sets of parameters. In all four, we simulate a population of 1000 individuals, each one represented by two bit-strings of 3200 bits size, with a mutation rate at birth of $m=1.0$. In case (a), $x=0.98$ (weak selection) and $p=0$, the environment does not change. The average genetic load starts at 0 (ideal individuals) and grows to a small value of order 1 . The distributions of genetic loads are shown in Figure 6, averaged after the initial 5000 time steps. For case (a), it has a peak at 0 , meaning that most of the population carries no genetic load, with a small width. When the environment changes with 

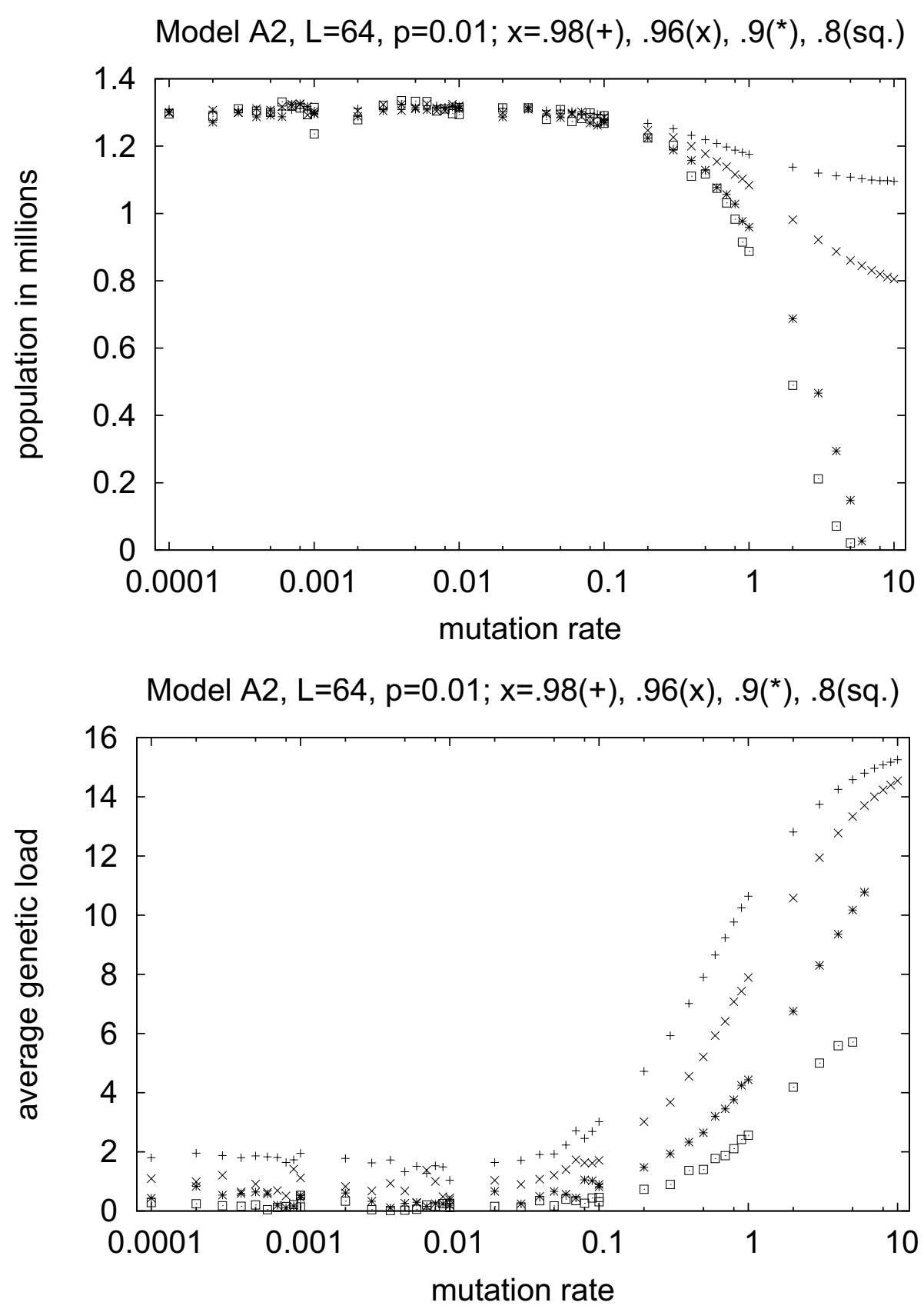

Fig. 3 - Model A2. As Figure 1 but with modified recessiveness and smaller $x$.

a probability of $p=0.01$ at each time step (case (b)), the average genetic load increases to a value of order 10 and its distribution peaks at a small non-zero value of the same order. Further increase in the rate of environmental change to $p=0.02$ leads the population to extinction (case (c)). The average genetic load increases rapidly and its distribution widens (Fig. 6). The genetic load accumulates thanks to the joint effects of the mu- tation rate at birth and a fast environmental change that, even with a weak selection, leads eventually to extinction. The need for a strong selection is now shown: for the same parameters $(p$ and $m$ ) but smaller $x=0.95$ (case (d)), the population resists and the distribution of genetic load is very similar to the one in case (b).

The same qualitative results were obtained for haploid asexual populations, but extinction is avoided only 
64 bits, $p=0.01, x=0.98$; deaths $(+), \operatorname{load}(x)$, perfects $\left({ }^{*}\right)$
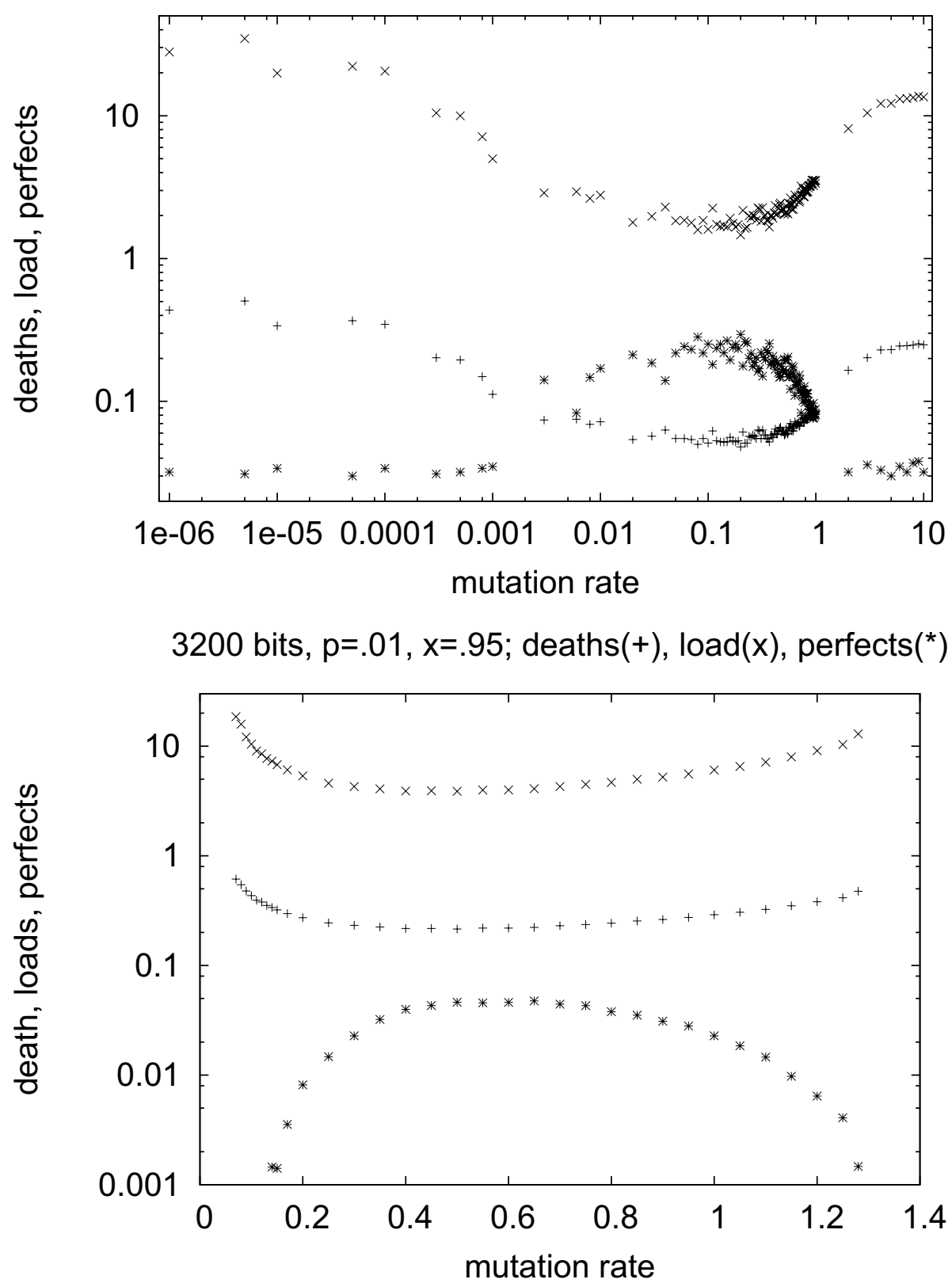

Fig. 4-Average genetic load $\langle N\rangle$, deaths and number of perfect individuals as a fraction of the population. Top figure for bit-strings of size 64 , and bottom figure for 3200 . Simulations running for $10^{6}$ time steps.

for larger populations and stronger selection pressure, similar to de Oliveira (2007b).

Extinction can then be correlated to features of the distribution of genetic load. It is avoided as long as the average genetic load is not much larger than the width of the distribution. This is more clearly shown in Figure 7, where we plot the results of simulations of populations with each individual represented by two bit-strings of 2048 bits size, with $x=0.9$ (strong selection), $m=1$, and $p$ ranged in the interval $[0,0.36]$. Both the average genetic load and the width of the distribution increase monotonically with $p$, while the frac- 


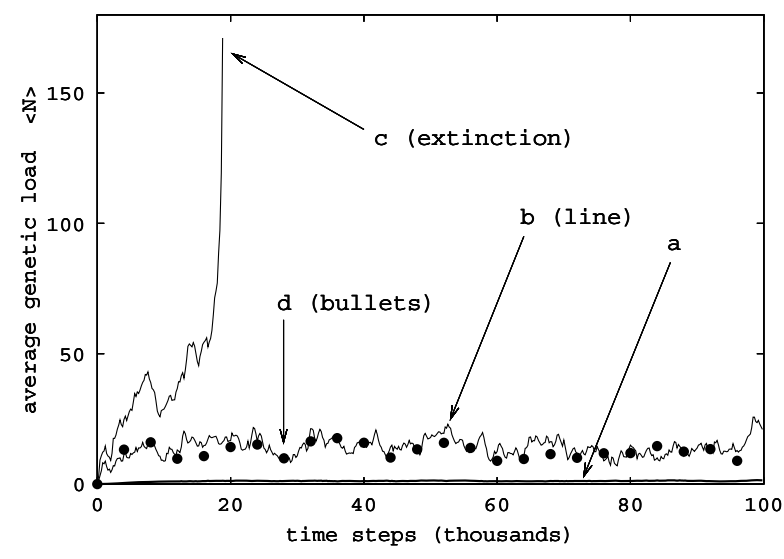

Fig. 5 - Time evolution of average genetic load for the four cases ((a)(d)) described in the text. Case (c) leads to extinction, while case (d) shows survival when selection is increased.

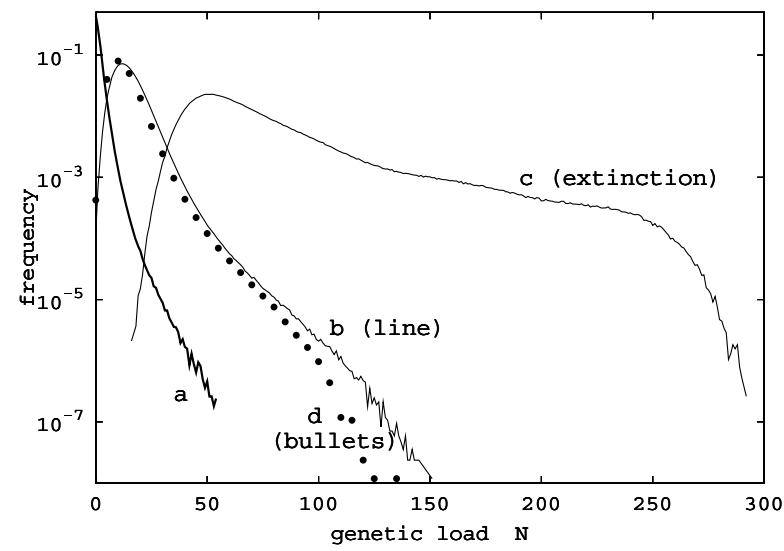

Fig. 6 - Distribution of genetic load for the four cases ((a)-(d)) described in the text.

tion of individuals with zero load decreases. Beyond $p=0.35$, this fraction vanishes and extinction is the outcome of the simulation. In the same plot, we also show the fraction of individuals that die (for genetic reasons only, in this model) at each time step. As $p$ increases, survival of the population becomes more difficult and causes this fraction to be ever increasing.

\section{CONCLUSION}

In all our models, the genetic heritage of a diploid individual is represented by a pair of bit-strings, which undergo mutations at birth, while the ideal phenotype is mapped into a single bit-string. Environmental change is translated into a mutation of this ideal phenotype. The genetic load of an individual is determined by a com-

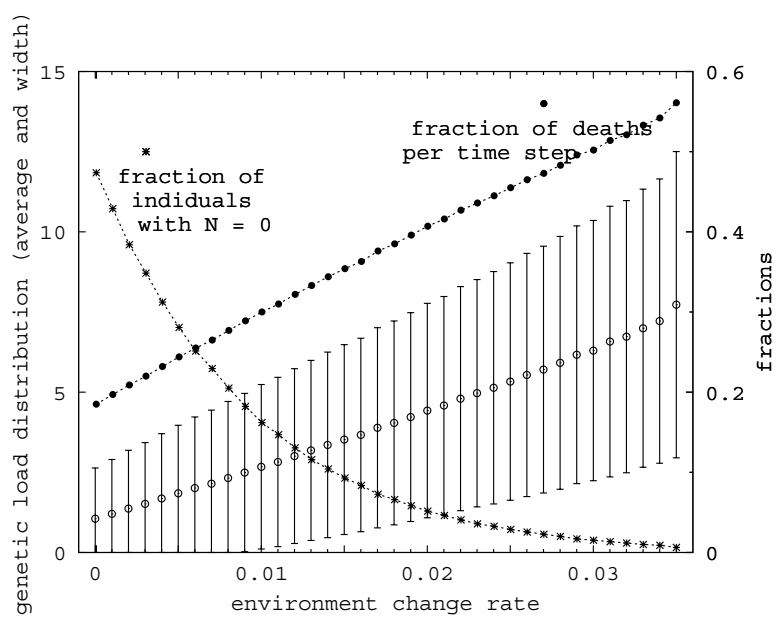

Fig. 7 - Average genetic load - open circles - and width of the distribution of load - error bars - (y scale on the left), fraction of the population with no genetic load and fraction of deaths per time step (y scale on the right). Values correspond to averages taken after 5000 initial time steps, up to $10^{6}$. Similar results are obtained for $L \leq 32768$.

parison between its genetic strings and the ideal phenotype. This genetic load determines the death probability of each individual.

Our results come from simulations with a fixed rate of environmental change and a fixed value for the parameter that measures selection strength $x$. We show that population fitness, determined by its size, reaches a broad maximum, while the average genetic load reaches a minimum, for some intermediate range of the mutation rate at birth (model A1). So, nature has self-organized its cellular error correction machinery to ensure a mutation rate within some range.

On the other hand, when the rate of environmental change increases, our results are consistent with the interpretation that selection has to get stronger to avoid population extinction (model B).

A more realistic approach would assign a different selective value for each different bit position, since different inherited diseases differ in their danger to survival. However, since these values would have to be free parameters, their introduction in the model would render it almost useless.

\section{ACKNOWLEDGMENTS}

The authors thank S. Cebrat for helpful comments on the manuscript. 


\section{RESUMO}

Simulação computacional de agentes individuais que se reproduzem e morrem por acúmulo de mutações herdadas mostra um máximo da população ou evita extinção, para taxas de mutação intermediárias. Assim, as mortes parecem necessárias para a evolução dos mais adaptados a um ambiente mutante.

Palavras-chave: ambiente mutante, evolução, modelagem computacional, mutação, seleção.

\section{REFERENCES}

Cebrat S And Pekalski A. 2004. The Role of Dominant Mutations in the Population Expansion. Lect Notes Comp Sci 3039: 765-770.

De OliveIRA PMC. 2001. Why Do Evolutionary Systems Stick to the Edge of Chaos? Theory Biosc 120: 1-19.

De Oliveira PMC. 2007a. A Importância das Flutuações em Biologia. Rev bras ens fis 29: 377-384.

De Oliveira PMC. 2007b. Chromosome Length Scaling in Haploid, Asexual Reproduction. J Phys CM19, 065147, $9 \mathrm{p}$.

De Oliveira PMC. 2009. Why Must We All Die? The Combined Role of Dissipation and Fluctuations in Evolutionary Biology. In: HiRTREITER C AND SCHNEIDER JJ (Eds), Lectures on Socio- and Econo-Physics, SpringerVerlag, Heidelberg.

De Oliveira PMC, Moss de Oliveira S, Bernardes AT AND Stauffer D. 1998. Siblings of Centenarians Live Longer: a Computer Simulation. The Lancet 352: 911-911.

De Oliveira PMC, Moss de Oliveira S, Bernardes AT And Stauffer D. 1999. Monte Carlo Simulations of Inherited Longevity. Physica A262: 242-248.
De Oliveira PMC, Moss de Oliveira S, Stauffer D, Cebrat S And Pekalski A. 2008. Does Sex Induce a Phase Transition? Eur Phys J B 63: 245-254.

KIRKWOOD TBL. 2005. Understanding the Odd Science of Aging. Cell 120: 437-447.

Moss de Oliveira S, DE Oliveira PMC ANd STAUFFER D. 1996. Ageing with Sexual and Asexual Reproduction: Monte Carlo Simulations of Mutation Accumulation. Braz J Phys 26: 626-630.

Moss de Oliveira S, De Oliveira PMC and StaufFER D. 1999. Evolution, Money, War and Computers. Teubner, Leibzig-Stuttgart.

PEnNA TJP. 1995. A Bit-String Model for Biological Aging. J Stat Phys 78: 1629-1633.

SÁ Martins JS AND Cebrat S. 2000. Random Deaths in a Computational Model for Age-Structured Populations. Theory Biosc 119: 156-165.

SÁ Martins JS And Moss de Oliveira S. 1998. Why Sex: Monte Carlo Simulation of Survival After Catastrophes. Int J Mod Phys C 9: 421-432.

Stauffer D. 2007. The Penna Model of Biological Aging. Bioinformatics and Biological Insights 1: 91-100 (www.la-press.com/article.php?article_id=520).

Stauffer D AND Cebrat S. 2006. Extinction in Genetic Bit-String Model with Sexual Reproduction. Adv Compl Syst 9: 147-156.

Stauffer D, De Oliveira PMC, Moss de Oliveira S, PEnNA TJP ANd SÁ Martins JS. 2001. Computer Simulations for Biological Asexual and Sexual Reproduction. An Acad Bras Cienc 73: 15-32.

Stauffer D, Moss de Oliveira S, De Oliveira PMC AND SÁ MARTins JS. 2006. Biology, Sociology, Geology by Computational Physicists, Elsevier, Amsterdam. 